Original Research Paper

\title{
Fluid-Structure Interaction Analysis of a Piezoelectric Cantilever for Energy Harvesting
}

\author{
Bader Alshuraiaan \\ Department of Mechanical Engineering, Kuwait University, P.O Box 5969, Safat 13060, Kuwait
}

Article history

Received: 01-06-2020

Revised: 04-07-2020

Accepted: 17-07-2020

Email:alshuraiaan@yahoo.com

\begin{abstract}
A numerical investigation was conducted to study the impact of varying the velocity magnitude and the elasticity of the cantilever on the performance of the piezoelectric beam such as tip deflection and voltage, tip electric field and maximum electric field. COMSOL software was used to solve for the governing equations taking into account fluid-structure interaction. The results of this investigation were compared against analytical results found in the literature and the agreement was excellent. The results presented in this study demonstrated a substantial impact of the flow velocity magnitude and elasticity of the cantilever on the tip deflection and voltage as well as maximum electric field. As the velocity increased and the elasticity of the microcantilever decreased, the tip deflection increased and consequently increased voltage and electric field. This study suggests that high voltage and electricity can be generated from energy harvester if they are subjected to high fluctuations.
\end{abstract}

Keywords: Piezoelectric, Fluid-Structure Interaction, Energy Harvesting, Microcantilever, Oscillating Structure, Fluid Flow

\section{Introduction}

The energy generated from oscillating structures has received a considerable attention by researchers in the last decades (Sonar and Paik, 2016; Cook-Chennault et al., 2008; Ghosh and Mandal, 2017; Liu et al, 2017; Ghosh et al., 2017; Lu et al., 2018; Curry et al, 2018; Markus and Hayes, 2017; Eggborn, 2003) due to its applications in various fields such as powering a remote sensor, wireless, autonomous and biomedical applications (Ghareeb et al., 2016). It was cited by many researchers that piezoelectric energy harvesters have many advantages such as low cost, flexibility and the fact that the required mechanical energy for conversion can be obtained from the surrounding environment. Umeda et al. (1996) studied Piezoelectric (PZT) materials and their applications in transforming the mechanical energy due to oscillations into electricity by a piezoelectric device. Beeby et al. (2006) reviewed the pertinent applications of piezoelectric structures in wireless devices. Lee et al. (2015) performed an experimental study on harvesting energy from internal flows using piezoelectric systems. Their results indicated that the energy produced $(20 \mathrm{~mW})$ from the proposed designs were identical using the same flow rate.

Many studies were conducted in the literature to analyze the performance of piezoelectric cantilever under various geometries and conditions to maximize its output power (Goldschmidtboeing and Woias, 2008; Benasciutti et al., 2010; Dietl and Garcia, 2010; Simon and Yves, 2009; Roundy, 2005). Goldschmidtboeing and Woias (2008) conducted a theoretical study based on Rayleigh-Ritz method to analyze triangular-shaped and rectangular-shaped beams for piezoelectric energy harvesters. Their results showed that triangular-shaped beams exhibited higher output power than rectangular beams. Simon and Yves (2009) investigated numerically the performance of different shapes of piezoelectric energy harvesters. Their illustrated that tapered beam with $0.3^{\circ}$ slope angle increased the collected energy by $69 \%$. Khanafer and Vafai (2018) investigated the effect of inlet velocity and thickness of the piezoelectric layer on heat transfer and voltage output. Their results show that the flow inlet velocity significantly enhances the both the heat transfer and voltage output of the piezoelectric material while increasing the thickness of the piezoelectric material decreases the voltage output from the system. Akaydin et al. (2010) conducted a numerical study to harvest energy from turbulent flow using piezoelectric cantilever. The piezoelectric cantilever was placed in the wake of a circular cylinder at high Reynolds numbers. The authors concluded that unsteady flow may exert oscillatory forces on the 
piezoelectric beam which can be used for harvesting energy. Akaydin et al. (2012) conducted an experimental study of a self-excited piezoelectric harvester connected to a cylinder subjected to an oscillatory force to cause deflection. An electrical power of $0.1 \mathrm{~mW}$ was produced at a speed of $1.192 \mathrm{~m} / \mathrm{s}$. Hobeck et al. (2014) conducted a numerical analysis using ANSYS-CFX to investigate dual cantilever flutter as related to energy harvesting. The two beams were subjected to a velocity of $6 \mathrm{~m} / \mathrm{s}$. The authors in that study claimed that the dual flutter beams had a potential application to harvest energy.

One can note from the above citations that many studies on energy harvester concentrated on rigid piezoelectric cantilever. Thus, the main objective of this work is to examine a the effect of flexible piezoelectric cantilever in harvesting energy at various relevant parameters such as flow velocity and elasticity of the microcantilever beam on the tip deflection and tip voltage, tip electric field and maximum electric field.

\section{Mathematical Formulation}

A two-dimensional piezoelectric microcantilever was analyzed in this investigation in Fig. 1. The flow was assumed incompressible and unsteady. An arbitrary Lagrangian-Eulerian formulation was implemented to solve the governing equation. The polarization $(\mathrm{P})$ of the piezoelectric structure is assumed in the positive $y-$ direction. Based on the above assumptions, the governing equations for this investigation can be summarized as follows (Khanafer and Vafai, 2018):

\section{Continuity}

$\nabla \cdot u=0$

\section{Momentum}

$\rho_{f} \frac{\partial u}{\partial t}+\rho_{f}(u-w) \cdot \nabla u=\nabla \cdot \sigma_{f}+f_{f}^{B}$

\section{Elastodynamics Equation}

$\rho_{s} \ddot{d}_{s}=\nabla \cdot \sigma_{s}^{\text {total }}+f_{s}^{B}$

The flow and interface velocity vectors are given by $u$ and $w$ respectively, $\rho_{f}$ and $\rho_{s}$ are the fluid and solid densities, $f_{f}^{B}$ and $f_{s}^{B}$ are the fluid and structure body forces per unit volume, respectively, $w$ is the moving mesh velocity. The fluid and structure stress tensors are given by: $\sigma_{f}$ and $\sigma_{s}$, respectively. The acceleration of the solid part is given by $\ddot{d}_{s}$. The boundary conditions are given by:

1. Inlet

$$
\text { At } x=0: u=U_{o}, v=0
$$

2. Exit

$$
\text { At } x=d: \frac{\partial u}{\partial x}=\frac{\partial v}{\partial x}
$$

3. Walls of the Channel

$$
\begin{aligned}
& y=0,0<x<d: u=v=0 \\
& y=H, 0<x<d: u=v=0
\end{aligned}
$$

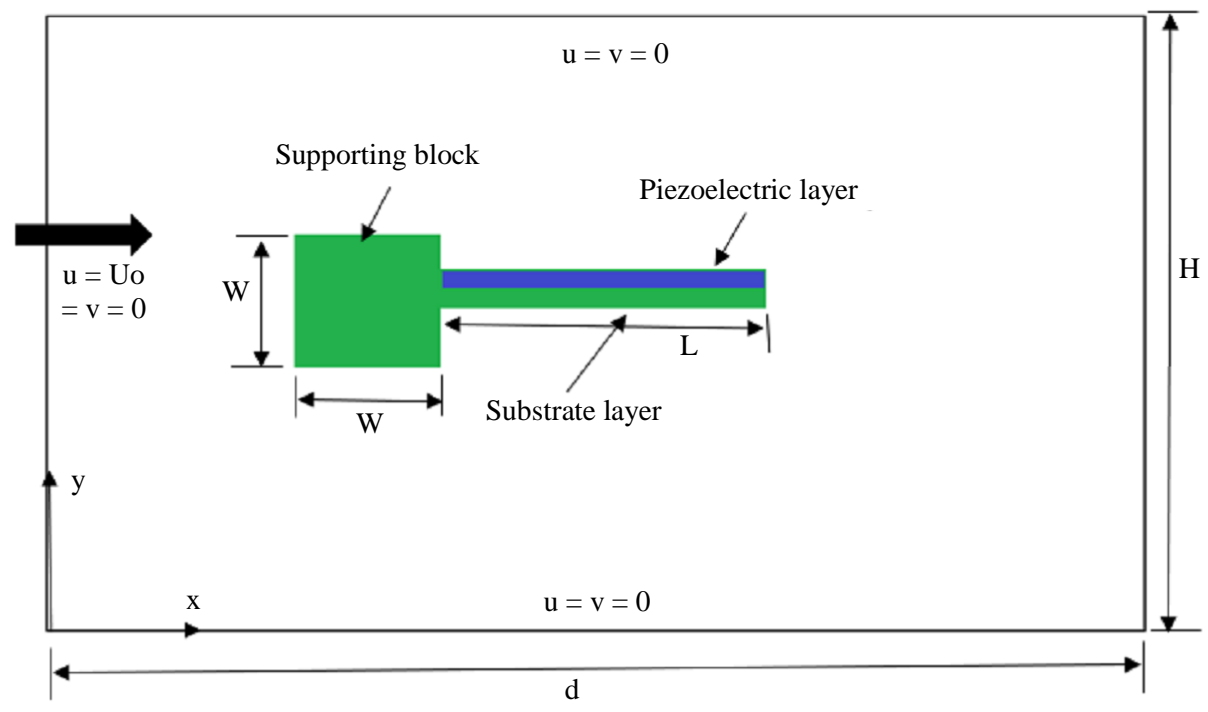

Fig. 1: Schematic of the model under investigation 
The interface boundary conditions between the two layers are summarized as follows:

$$
\text { Displacement } \quad d_{f}=d_{s}
$$

Traction $\quad \sigma_{f}=\sigma_{s}$

where, $d_{f}$ is the displacement of the fluid domain and $d_{s}$ is the displacement of the solid structure. The mechanical properties of a flexible microcantilever beam resembles those of rubber $\left(v=0.35, \rho_{s}=2000 \mathrm{~kg} / \mathrm{m}^{3}, E=\right.$ $=0.25 \mathrm{MPa}) . E$ represents the elasticity of the beam while $v$ represents the Poisson's ratio. The physical properties of the piezoelectric structure are given by (Khanafer and Vafai, 2018):

$$
\begin{aligned}
& E=61 G P a, G=21.1 G P a, v=0.35 \\
& e_{x y}=-7.2, e_{y y}=15.1, e_{5 x}=12.3 \\
& \varepsilon_{x x}=1.53 \times 10^{-8}, \varepsilon_{y y}=1.53 \times 10^{-8}
\end{aligned}
$$

\section{Numerical Scheme}

COMSOL (v5.2) software based finite element formulation was utilized in this investigation The Newton-Raphson method was utilized in this study to solve the discretized. A convergence criterion of $1 \times 10^{-4}$ was used in this study. Adaptive mesh refinement with extremely fine mesh around the beam is used using COMSOL to ensure grid independence throughout the study. Initial conditions of zero velocity and zero deflection are set for this study.

\section{Model Validation}

The present results were compared against analytical solution of deflection reported in the literature by (Smits et al., 1991). as follows:

$$
\delta(x, V)=-\frac{3 d_{31} V x^{2}}{8 t_{p}^{2}}
$$

where, $V$ is the applied voltage, $t_{p}$ is the thickness of piezoelectric beam and $d_{31}$ is the unclamped transverse piezoelectric coefficient. Figure 2 demonstrates an excellent comparison of the deflection along the beam between the present results and the analytical results of (Smits et al., 1991).

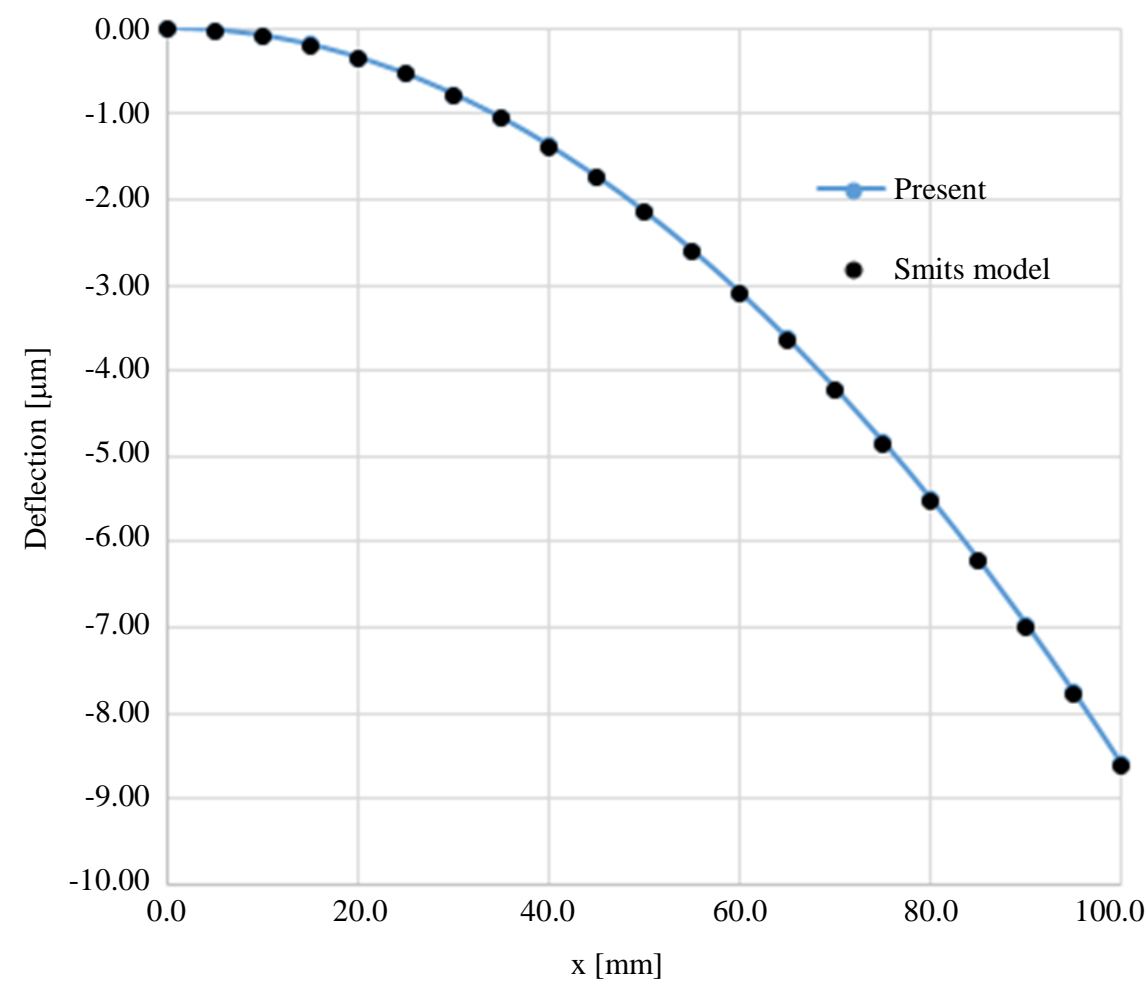

Fig. 2: Deflection along the beam between the present results and that of Smits et al. $(1991)\left(V=100\right.$ volt, $t_{p}=1 \mathrm{~mm}, d_{31}=2.3 \times 10^{-11}$ $\mathrm{C} / \mathrm{N})$ 


\section{Results and Discussion}

The effect of changing various pertinent parameters on the performance of the piezoelectric beam was analyzed in this investigation. It can be seen from Fig. 3 that the tip deflection fluctuates in a harmonic shape for various inlet velocities. As the velocity of the inlet flow rises, the tip deflection of the piezoelectric beam enhances significantly due to larger inertia forces acting on the beam. This effect is noticeable at an inlet flow velocity of $1.5 \mathrm{~m} / \mathrm{s}$. Figure 4 shows the effect of changing the inlet velocity on the tip voltage. The tip deflection increases with an increase in the flow velocity and consequently increases the tip voltage. Figure 4 shows that the tip voltage oscillates in a harmonic pattern for low velocity. However, for higher velocities, the tip voltage increases and oscillates more randomly and not in a sinusoidal mode.

The effect of varying the inlet velocity has a substantial impact on the tip electric field is shown in Fig. 5. Figure 5 demonstrates that the tip electric field increases significantly with an increase in the velocity. The electric field is directly proportional with the voltage. Figure 6 illustrates the variation of the maximum electric field with the inlet flow velocity. The present results indicated that the maximum electric field was found near the supporting mechanism because the bending moment is maximum at that location. Figure 6 shows that the maximum electric field increases with an increase in the inlet velocity.

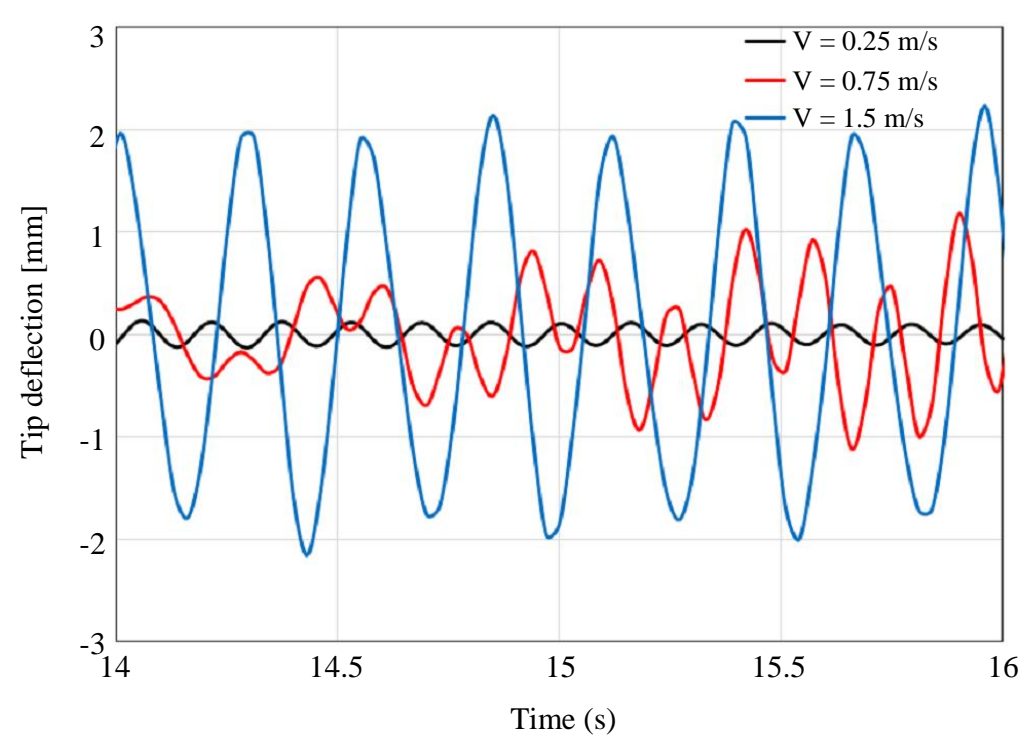

Fig. 3: Tip deflection for various inlet flow velocity

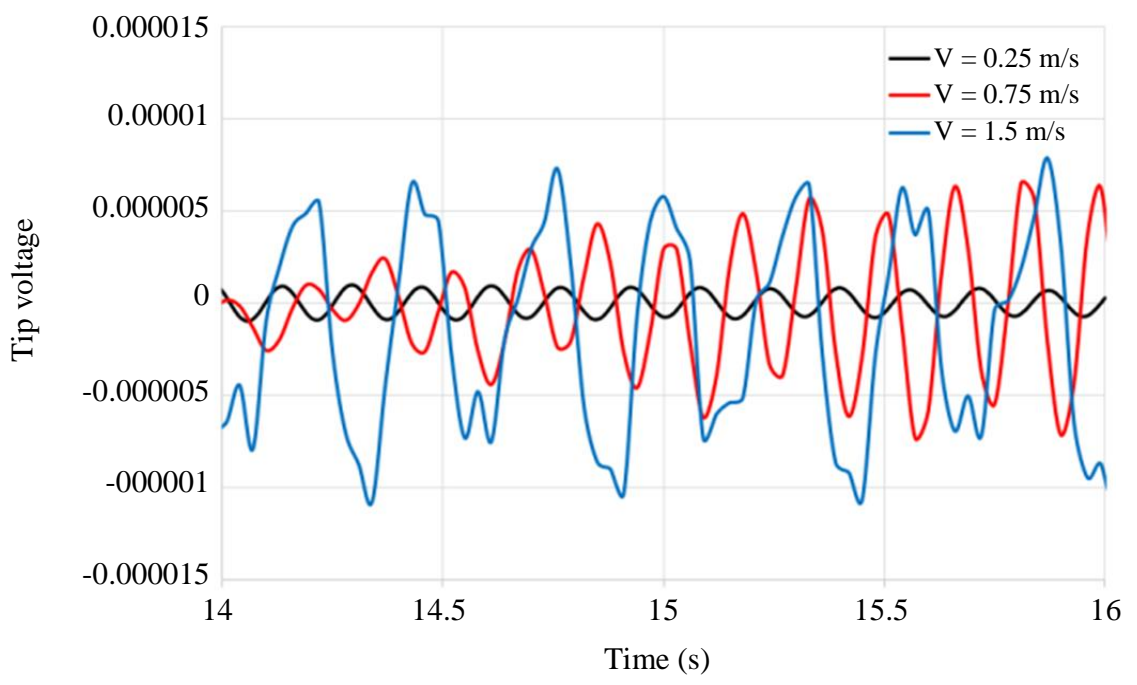

Fig. 4: Tip voltage of the piezoelectric cantilever for various inlet flow velocity 
Bader Alshuraiaan / American Journal of Engineering and Applied Sciences 2020, 13 (3): 368.374 DOI: 10.3844/ajeassp.2020.368.374

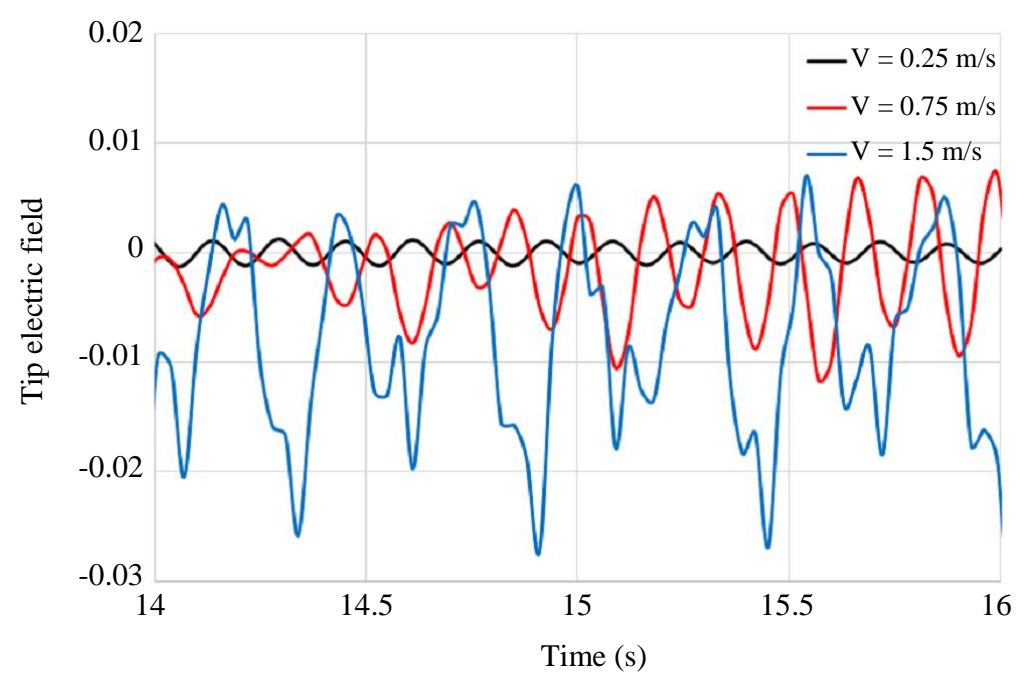

Fig. 5: Tip electric field for various inlet flow velocity

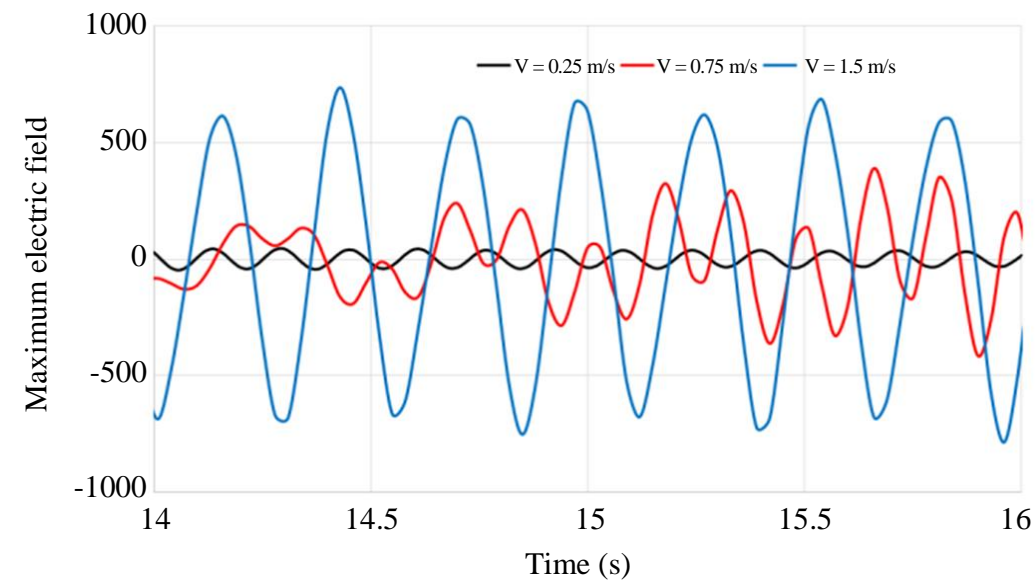

Fig. 6: Maximum electric field variation vs. inlet velocity

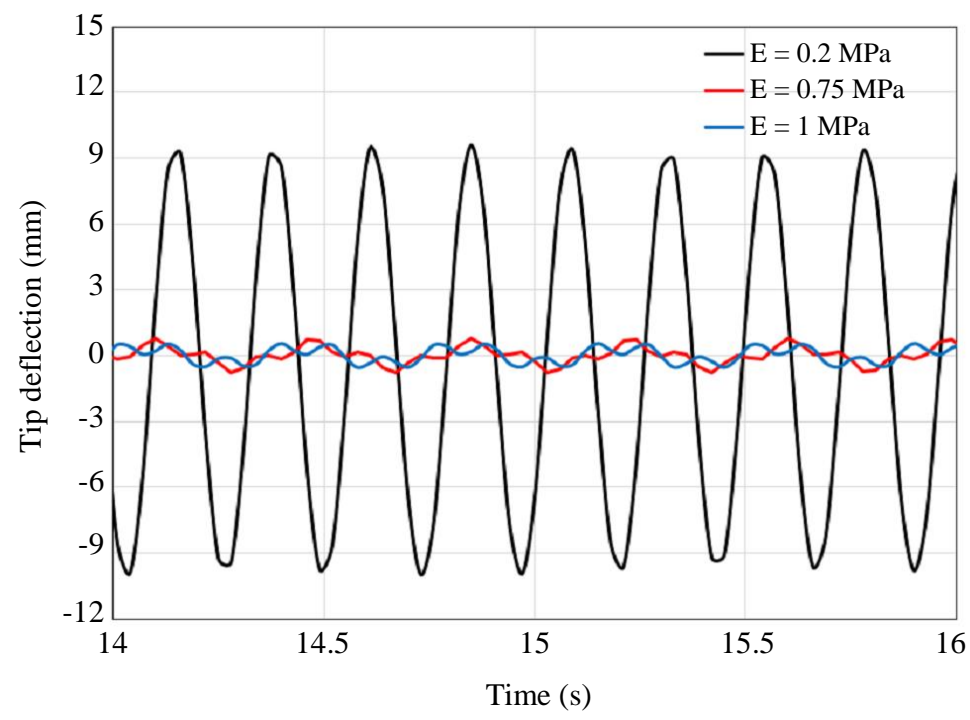

Fig. 7: Tip deflection of the piezoelectric cantilever vs. elasticity 


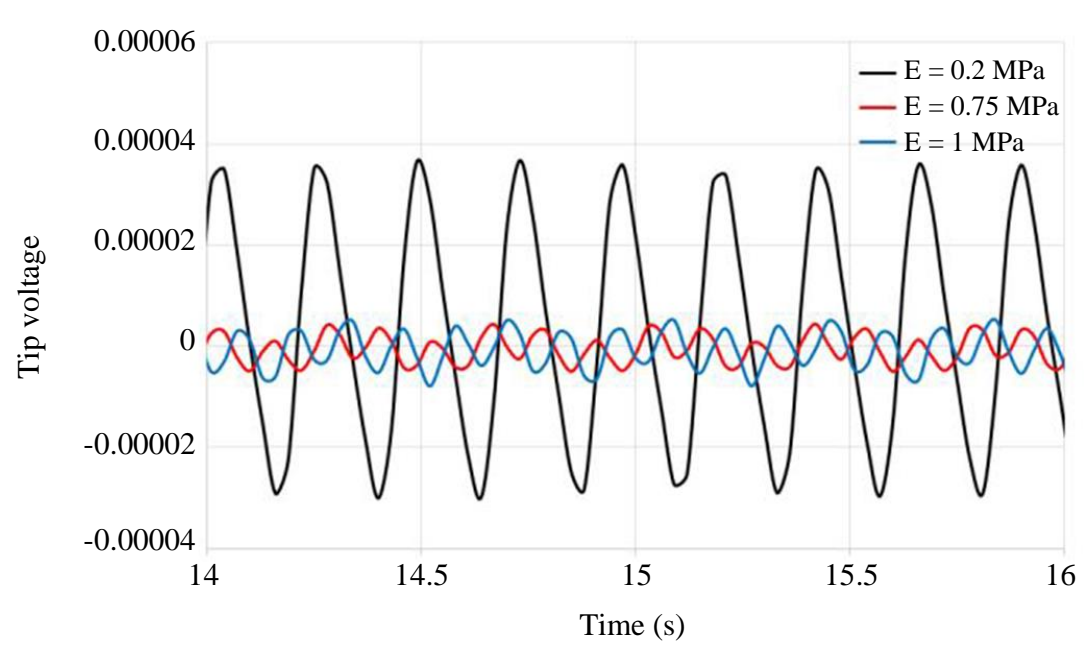

Fig. 8: The tip voltage of the piezoelectric cantilever vs. elasticity

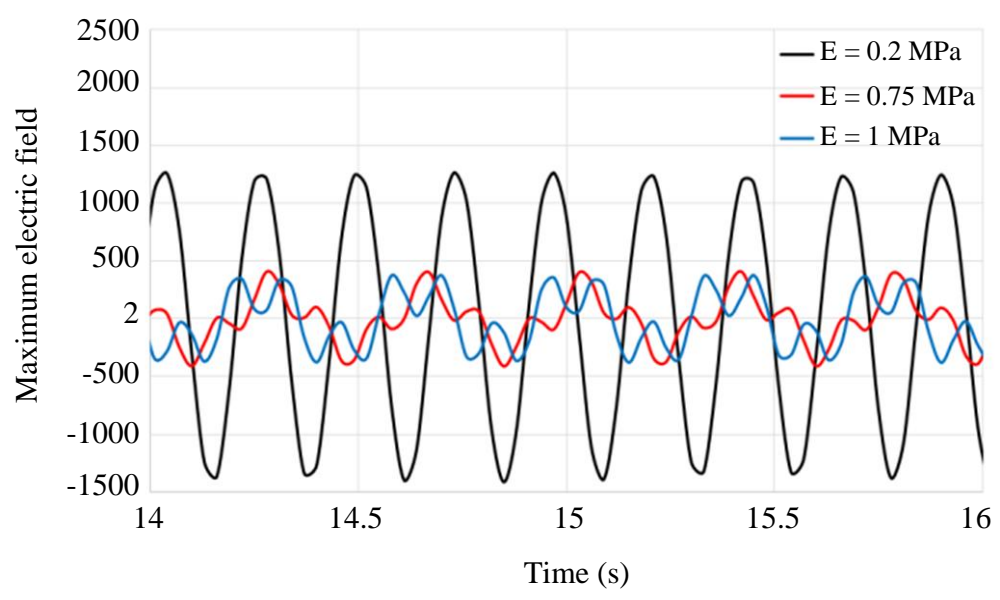

Fig. 9: Maximum electric field of the piezoelectric cantilever vs. elasticity

Figures 7 through 9 demonstrate the effect of changing the elastic modulus of the cantilever on the tip deflection, tip voltage and maximum electric field. These figures clearly show that the modulus of elasticity of the cantilever has a significant effect on these parameters. Figure 7 shows that the tip deflection increases significantly with a decrease in the elasticity of the cantilever $(\mathrm{E}=0.2 \mathrm{MPa})$ and consequently increases the tip voltage as depicted in Fig. 8. The effect of changing the elasticity of the cantilever on the maximum electric field of the piezoelectric cantilever is represented in Fig. 9. As the elasticity of the cantilever decreases, the maximum electric field improves significantly for $\mathrm{E}=0.2 \mathrm{MPa}$.

\section{Conclusion}

The characteristics of piezoelectric beam was analyzed in this study for various pertinent parameters such as flow velocity and elasticity of the cantilever beams. The results of the present investigation showed that the value of the inlet flow velocity had considerable effects on the tip deflection and voltage. As the inlet velocity increased, the tip deflection increased and subsequently increased the generated voltage. Moreover, tip deflection was found to increase considerably with a reduction in the elasticity of cantilever and consequently increasing tip voltage and maximum electric field.

\section{Funding Information}

This work was supported and funded by Kuwait University, Research Project No. (EM01/17).

\section{Ethics}

Author confirms that they are no ethical issues and no conflict of interest that may arise after the publication of this manuscript. 


\section{References}

Akaydin, H. D., Elvin, N., \& Andreopoulos, Y. (2010). Energy harvesting from highly unsteady fluid flows using piezoelectric materials. Journal of Intelligent Material Systems and Structures, 21(13), 1263-1278.

Akaydin, H. D., Elvin, N., \& Andreopoulos, Y. (2012). The performance of a self-excited fluidic energy harvester. Smart materials and Structures, 21(2), 025007.

Beeby, S. P., Tudor, M. J., \& White, N. M. (2006). Energy harvesting vibration sources for microsystems applications. Measurement science and technology, 17(12), R175.

Benasciutti, D., Moro, L., Zelenika, S., \& Brusa, E. (2010). Vibration energy scavenging via piezoelectric bimorphs of optimized shapes. Microsystem technologies, 16(5), 657-668.

Cook-Chennault, K. A., Thambi, N., \& Sastry, A. M. (2008). Powering MEMS portable devices-a review of non-regenerative and regenerative power supply systems with special emphasis on piezoelectric energy harvesting systems. Smart materials and structures, 17(4), 043001.

Curry, E. J., Ke, K., Chorsi, M. T., Wrobel, K. S., Miller, A. N., Patel, A., ... \& Kuo, C. L. (2018). Biodegradable piezoelectric force sensor. Proceedings of the National Academy of Sciences, 115(5), 909-914.

Dietl, J. M., \& Garcia, E. (2010). Beam shape optimization for power harvesting. Journal of Intelligent Material Systems and Structures, 21(6), 633-646.

Eggborn, T. (2003). Analytical models to predict power harvesting with piezoelectric materials (Doctoral dissertation, Virginia Tech).

Ghareeb, N., Gaith, M., Alloush, T., \& Al-Sarraf, M. (2016). Energy Harvesting from Vibrating PiezoElectric Structures. Journal of Robotics and Mechanical Engineering Research, 1(3), 14-21.

Ghosh, S. K., \& Mandal, D. (2017). Bio-assembled, piezoelectric prawn shell made self-powered wearable sensor for non-invasive physiological signal monitoring. Applied Physics Letters, 110(12), 123701 .

Ghosh, S. K., Adhikary, P., Jana, S., Biswas, A., Sencadas, V., Gupta, S. D., ..., \& Mandal, D. (2017). Electrospun gelatin nanofiber based self-powered bio-e-skin for health care monitoring. Nano Energy, 36, 166-175.

Goldschmidtboeing, F., \& Woias, P. (2008). Characterization of different beam shapes for piezoelectric energy harvesting. Journal of micromechanics and microengineering, 18(10), 104013.
Hobeck, J. D., Geslain, D., \& Inman, D. J. (2014, April). The dual cantilever flutter phenomenon: a novel energy harvesting method. In Sensors and Smart Structures Technologies for Civil, Mechanical and Aerospace Systems 2014 (Vol. 9061, p. 906113). International Society for Optics and Photonics.

Khanafer, K., \& Vafai, K. (2018). Analysis of heat transfer and flow characteristics of a microcantilever beam for piezoelectric energy harvesting. International Communications in Heat and Mass Transfer, 98, 265-272.

Lee, H. J., Sherrit, S., Tosi, L. P., Walkemeyer, P., \& Colonius, T. (2015). Piezoelectric energy harvesting in internal fluid flow. Sensors, 15(10), 26039-26062.

Liu, Z., Zhang, S., Jin, Y. M., Ouyang, H., Zou, Y., Wang, X. X., ..., \& Li, Z. (2017). Flexible piezoelectric nanogenerator in wearable selfpowered active sensor for respiration and healthcare monitoring. Semiconductor Science and Technology, 32(6), 064004.

Lu, C., Wu, S., Lu, B., Zhang, Y., Du, Y., \& Feng, X. (2018). Ultrathin flexible piezoelectric sensors for monitoring eye fatigue. Journal of Micromechanics and Microengineering, 28(2), 025010.

Markus, D. T., \& Hayes, M. C. (2017). U.S. Patent No. 9,632,333. Washington, DC: U.S. Patent and Trademark Office.

Roundy, S. (2005). On the effectiveness of vibrationbased energy harvesting. Journal of intelligent material systems and structures, 16(10), 809-823.

Simon, P., \& Yves, S. A. (2009). Electromechanical performances of different shapes of piezoelectric energy harvesters. international workshop smart materials and structures.

Smits, J. G., Dalke, S. I., \& Cooney, T. K. (1991). The constituent equations of piezoelectric bimorphs. Sensors and Actuators A: Physical, 28(1), 41-61.

Sonar, H. A., \& Paik, J. (2016). Soft pneumatic actuator skin with piezoelectric sensors for vibrotactile feedback. Frontiers in Robotics and AI, 2, 38.

Umeda, M., Nakamura, K., \& Ueha, S. (1996). Analysis of the transformation of mechanical impact energy to electric energy using piezoelectric vibrator. Japanese Journal of Applied Physics, 35(5S), 3267. 